\title{
Avalanches and Slip Patterning in Plastic Deformation
}

\author{
M. Zaiser ${ }^{1,2, *}$ and E. C. Aifantis ${ }^{2,3}$ \\ ${ }^{1}$ The University of Edinburgh, Center for Materials Science and Engineering, \\ The King's Buildings, Sanderson Building, Edinburgh EH93JL, UK \\ ${ }^{2}$ Aristotle University of Thessaloniki, Polytechnic School, \\ 54006 Thessaloniki, Greece \\ ${ }^{3}$ Michigan Technological University, Houghton, MI 47931, USA
}

\begin{abstract}
In materials deforming in planar slip, it has been observed that plastic deformation proceeds in discrete 'avalanches' the sizes of which obey power-law statistics. In the spatial domain, these avalanches produce patterns of mesoscopic deformation in the form of slip lines and slip bands. This type of mesoscopically jerky and localized plastic flow is observed in spite of a globally convex stress-strain relationship. It is shown that avalanche dynamics of plastic flow can be modelled by a generalization of linear viscoplastic constitutive models which includes (i) randomness in the local hardening law and (ii) a spatial coupling described by a second-order gradient term as previously used in modelling macroscopic slip localization and shear band morphology in materials with non-convex stress-strain laws. It is demonstrated that the model accounts for the observation of slip avalanches as well as for the associated surface morphology. Effects of specimen size and external driving mode (machine stiffness, driving rate) on the avalanche dynamics are predicted and it is shown that in the limit of zero hardening the system exhibits features of self-organized critical behaviour.
\end{abstract}

Keywords: Slip Localization, Avalanches, Self-Organized Criticality, Constitutive Modeling.

\section{INTRODUCTION}

The characterization of plastic instabilities and associated deformation localization phenomena has been a challenge for the development of constitutive models for many years. The necessity to formulate constitutive equations which are mathematically well-posed in these situations has led to the development of gradient

\footnotetext{
"Corresponding author: E-mail address: M.Zaiser@ed.ac.uk; Tel: -44-131-6505671; Fax: -44-131-6673677
} 
models /1-3/. From the viewpoint of materials science, the introduction of gradient terms into macroscopic constitutive equations is necessitated by the fact that the loss of macroscopic stability of deformation unveils the presence of internal length scales in the microstructure of deforming materials: The non-analytic behaviour of local constitutive models in case of instability is removed by the non-local couplings which are present on mesoscopic and microscopic scales. The relation between gradient terms and internal microstructural length scales has been demonstrated in many cases. An example is the proportionality between shear band widths and grain sizes in polycrystals $13 /$. While, depending on materials and deformation conditions, spatial couplings may be governed by different mechanisms (for an overview, see e.g. /4/), it is now generally accepted that the introduction of gradient terms in the constitutive equations is an adequate and physically motivated means of dealing with the problem of deformation localization in unstable plastic flow.

However, deformation heterogeneity and slip localization are not necessarily associated with macroscopically unstable deformation. On the microscopic scale, slip localization is a trivial consequence of the discreteness of defects. For instance, in crystalline solids the Burgers vectors of dislocations which carry the plastic deformation define discrete 'quanta' of slip. Slip localization phenomena which take place in spite of macroscopically stable deformation may also be observed on mesoscopic scales where they involve the collective dynamics of very large numbers of elementary defects. A classical example is the formation of slip lines or slip bands in crystalline solids deforming by planar slip. The slip steps on the surface of these materials manifest the collective motion of large numbers of dislocations $/ 5 /$ and a very high degree of deformation localization, in spite of the fact that macroscopic deformation is smooth and homogeneous. It has been shown by a number of authors that the distribution of slip lines and the general surface morphology in these materials obey fractal statistics /6-9/.

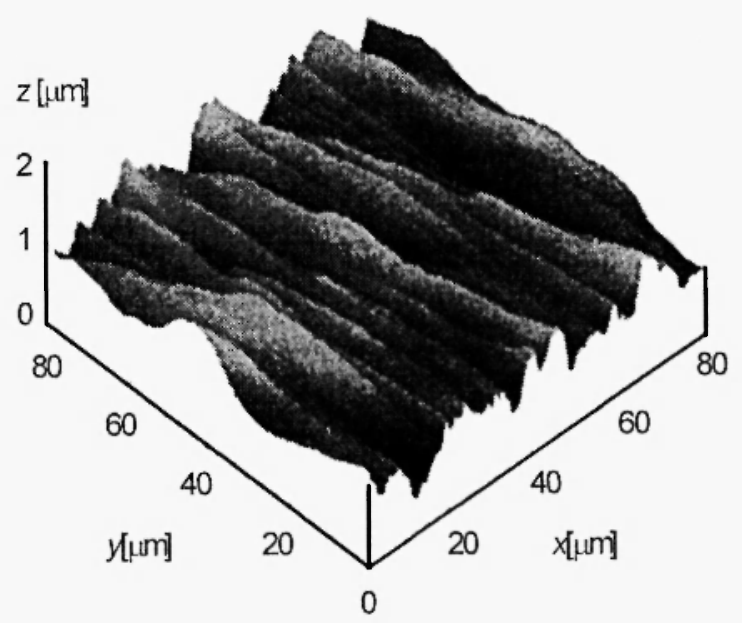

Fig. 1: SEM micrograph of the surface of a deformed Ni single crystal, after Meißner et al. /9/ 


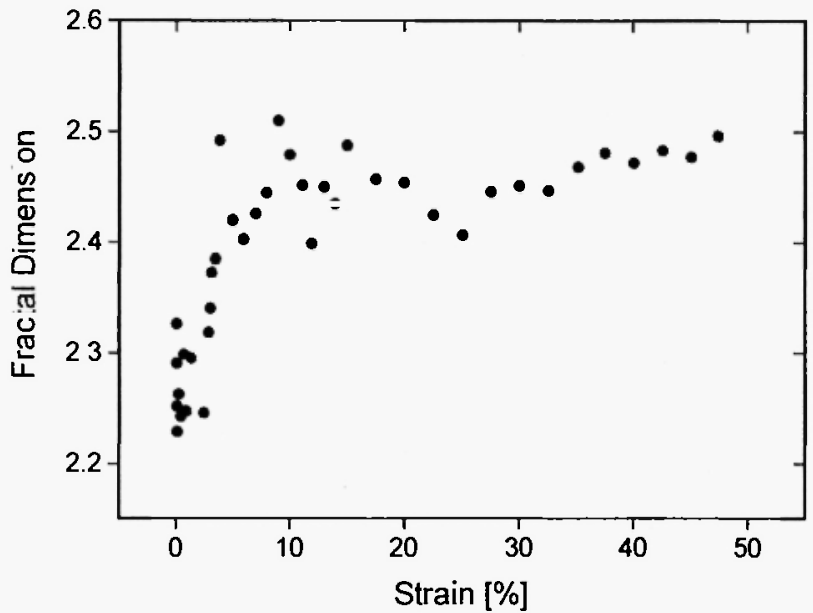

Fig. 2: Evolution of the fractal dimension of the surface of a Ni single crystal in the course of deformation

Heterogeneity of slip in spite of macroscopically stable deformation is illustrated in Figures 1 and 2. Figure 1 shows a scanning electron micrograph of the surface of a $\mathrm{Ni}$ single crystal deformed into hardening stage II (after Meißner et al. /9/). The surface exhibits a lamellar morphology; the direction of the lamellae (the $y$ direction in Figure 1) corresponds to the intersection of the surface with the slip plane. Normal to this direction, the surface exhibits a rough random profile. Such random surfaces can be characterized in terms of their fractal dimension /10/. The fractal dimension of the surface of a deforming $\mathrm{Ni}$ single crystal was determined by Meißner et al. from such micrographs taken at different strains. It was found that the fractal dimension increases during a strain interval of a few percent from two (flat surface) towards a constant value of about 2.5 (Figure 2).

While slip line and surface observations reveal information about the spatial organization of slip, information about the temporal dynamics of plastic flow can be obtained from acoustic emission measurements $/ 11,12 /$. Measurements performed by Weiss et al. /II/ on ice single crystals indicate an intermittent noise signal composed of discrete 'events'. The event sizes obey power-law statistics (Figure 3): the probability density to observe events with energy release $E$ decreases according to $p(E) \sim E^{\text {r }}$ where $\tau \approx$ 1.6. This observation indicates that plastic deformation proceeds in avalanches with a power-law size distribution reminiscent of the power-law distributions observed in many systems close to critical points.

The present paper is devoted to an extension of macroscopic constitutive models in order to describe spontaneous localization and avalanche dynamics of plastic deformation. To describe inhomogeneity and jerkiness of plastic flow in spite of a convex macroscopic stress-strain law, it is necessary to allow for local deviations from the macroscopic behaviour. This has been known for a long time in slip line theory, where one has to assume some local softening mechanism which is responsible for the initiation of a slip line. In the present work, we envisage the interplay between local softening and hardening processes in terms of statistical fluctuations in the local flow stresses. In this respect the present model can be envisaged as a phenomenological generalization of stochastic models of plastic flow as proposed during the last years by 


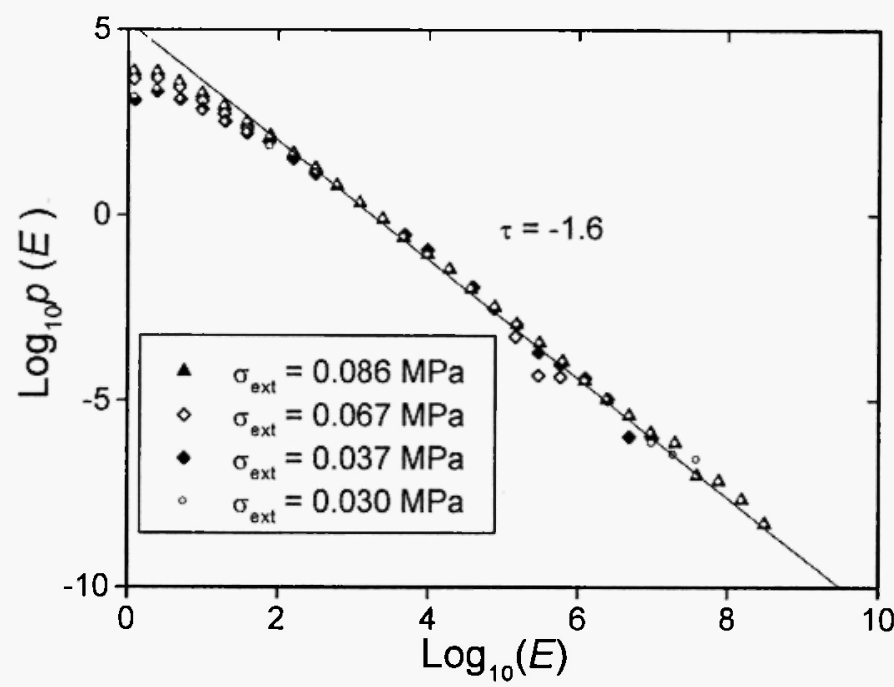

Fig. 3: Distribution of energy releases in acoustic emission 'events' recorded during creep deformation of ice single crystals; temperature $T=263 \mathrm{~K}$, resolved shear stresses on the basal plane as indicated in the inset. Over several orders the data follow a power law $p(E) \sim E^{-16}$. After Miguel et al. /12/.

Hähner et al. $/ 13,14 /$. While fluctuations in the flow stress (which can be related to inhomogeneities in the microstructure of the material) tend to make it deform heterogeneously on small scales, the regularizing character of the global hardening tends to smooth out the fluctuations on larger scales. By introducing a spatial coupling in the form of a gradient term one can provide for an additional interaction between adjacent volume elements which leads to spatial correlations in the slip pattern and to an avalanche-like dynamics. Plastic deformation in this case exhibits substantial spatio-temporal fluctuations on mesoscopic scales. It is shown in the following that the dynamics in this case exhibits strong analogies with physical systems which exhibit so-called depinning transitions and, in the particular case of zero hardening, displays features of selforganized criticality (SOC) $/ 15 /$.

\section{FORMULATION OF THE MODEL}

\subsection{Constitutive equations}

We consider a material deforming in pure shear along a single slip plane and slip direction. Without loss of generality, we identify the slip plane with the $y z$ plane and the slip direction with the $y$ direction of a Cartesian coordinate system. The rate of slip $\partial_{1} \gamma$ is assumed to be governed by a linear viscoplastic constitutive relationship,

$$
\mu \partial, \gamma=\sigma_{\mathrm{EXT}}-\sigma_{\mathrm{INT}}(\gamma \cdot x)
$$


where $\sigma_{\mathrm{EXT}}$ is the externally applied stress resolved in the slip direction (i.e., the $x y$-component of the externally applied stress) and $\sigma_{\mathrm{INT}}(\gamma, x)$ is a local internal stress (deformation resistance) which is a function of local strain. The external stress is assumed constant in space. Under the considered deformation conditions, heterogeneities of slip in the directions parallel to the slip plane necessarily lead to large incompatibility stresses, while heterogeneities of slip normal to the slip plane are stress-free. Because of this, one can in a first approximation assume that slip is homogeneous in the $y z$ plane and, hence, the local shear $\gamma=\gamma(x)$ is a function of the $x$ coordinate only. Rate-independent plasticity is recovered from Eq. (1) by letting the viscoplastic coefficient $\mu$ go to zero.

We assume that the local deformation resistance is made up by three contributions,

$$
\sigma_{\mathrm{INT}}(\gamma, x)=\bar{\sigma}_{\mathrm{INT}}(\gamma)+\delta \sigma(\gamma, x)-c \frac{\partial y^{2}}{\partial x^{2}}
$$

A detailed derivation of the constitutive model defined by Eqs. (1) and (2) from the dynamics of discrete dislocations will be given elsewhere /16/. The first term on the right-hand side of Eq. (2) equation has deterministic character. In the present study, we assume for simplicity linear hardening with a constant hardening coefficient $\theta$, i.e.

$$
\bar{\sigma}_{\mathrm{INT}}(\gamma)=\sigma_{0}+\theta \gamma
$$

where $\sigma_{0}$ is the critical resolved shear stress at the beginning of deformation. The second term on the righthand side of Eq. (2) is a fluctuating internal-stress contribution with fixed statistical properties. The autocorrelation function of $\delta \sigma(y, x)$ is assumed in the form

$$
\left\langle\delta \sigma(\gamma, x) \delta \sigma\left(\gamma^{\prime}, x^{\prime}\right)\right\rangle=\left\langle\delta \sigma^{2}\right\rangle f\left(\frac{x-x^{\prime}}{\xi}\right) g\left(\frac{\gamma-\gamma^{\prime}}{\gamma_{\mathrm{CORR}}}\right),
$$

where the functions $f$ and $g$ are normalized such that $f(0)=g(0)=1$ and $\int f(s) \mathrm{d} s=\int g(s) \mathrm{d} s=1$. In Eq. (4), $\left\langle\delta \sigma^{2}\right\rangle$ defines the 'amplitude' of the flow stress variations, $\gamma_{\text {CORR }}$ is the characteristic strain interval over which such fluctuations persist, and $\xi$ is the characteristic range over which $\delta \sigma(\gamma, x)$ changes in space. Finally, the third term on the right-hand side of Eq. (2) describes a strain-gradient dependent stress contribution which can be related to the presence of finite-range correlations in the dislocation patterns which are responsible for the deformation resistance. (For a detailed derivation see, again, /16/).

In the following it is convenient to use non-dimensional variables. Scaling the space coordinate by the correlation length $\xi$, time by $\mu \xi^{2} / c$, strain by the characteristic strain $\gamma_{\text {CORR }}$, and stress by $c \gamma_{C O R R} / \xi^{2}$, we obtain from Eqs. (1)-(3) 


$$
\dot{Y}(x)=S-S_{0}-H Y(X)+W(Y, X)+Y_{X X} .
$$

Here $Y=\gamma / \gamma_{\text {CORR }}$ is the scaled strain, $X=x / \xi$ the scaled space coordinate, $S=\xi^{2} \sigma_{\mathrm{EXT}} /\left(c \gamma_{\mathrm{CORR}}\right)$ the scaled external stress, $S_{0}$ the scaled initial flow stress, and $H=\xi^{2} \theta / c$ the scaled hardening coefficient. The scaled flow stress fluctuation $W$, finally, is a random field with zero average, mean square $\left\langle W^{2}\right\rangle=\left\langle\delta \sigma^{2}\right\rangle \xi^{4}$ $/\left(c \gamma_{\text {CORR }}\right)^{2}$, and unit correlation length.

\subsection{External driving: machine equation}

The response of systems which exhibit avalanche dynamics often depends on the mode of driving. In the following we consider deformation in strain rate control where the total (elastic plus plastic) strain rate $\dot{\gamma}_{\mathrm{EXT}}$ is prescribed externally. The temporal evolution of the external stress is then given by $\partial_{1} \sigma_{\mathrm{EXT}}=m\left(\dot{\gamma}_{\mathrm{EXT}}-\{\partial, \gamma(x)\}\right)$ where $m$ is the effective elastic modulus of the specimen + machine system and the curved brackets denote the spatial average over the specimen length $L,\left\{\partial_{1} \gamma(x)\right\}=(1 / L) \int \partial, \gamma(x) \mathrm{d} x$. Time integration yields

$$
\sigma_{\mathrm{EXT}}=m\left(\gamma_{\mathrm{EXT}}-\{\gamma(x)\}\right], \text { or, in scaled variables, } S=M\left(Y_{\mathrm{EXT}}-\{Y\}\right)
$$

where the scaled machine stiffness $M=m \xi^{2} / c$.

\section{ANALYSIS OF THE MODEL}

\subsection{Behaviour in the absence of spatial couplings}

In our model, the fluctuating term $\delta \sigma(\gamma, x)$ in Eq. (2) is responsible for the local softening which is required for slip-line formation. The local stress-strain characteristics which result from the superposition of the first and second terms on the right-hand side of Eq. (2) are schematically illustrated in Figure 4 . When spatial couplings are absent ( $c=0$ in Eq. (2)), deformation is locally characterized by 'jumps' across the softening regions; each of these jumps corresponds to the formation of a slip line. Since correlations in $\delta \sigma(\gamma, x)$ are restricted to distances $\sim \xi$, on larger scales these jumps proceed in a statistically independent manner. Therefore, fluctuations average out over the specimen length $L$ (the effective amplitude of the macroscopic flow-stress fluctuations is of the order of $\delta \sigma \sqrt{\xi / L}$ ) and, hence, deformation is smooth and homogeneous on large scales. The only noticeable effect of the randomness introduced by the term $\delta \sigma(\gamma, x)$ in this case is to induce (i) a roughening of the surface due to the small-scale randomness of the slip process (cf. the discussion below in Section 4), and (ii) an increase in the effective flow stress by approximately $\left\langle\delta \sigma^{2}\right\rangle^{1 / 2}$. This is due to the fact that the flow stress is governed by the stress required to move the system over the 'humps' in the local stress-strain characteristics, see Figure 4. 


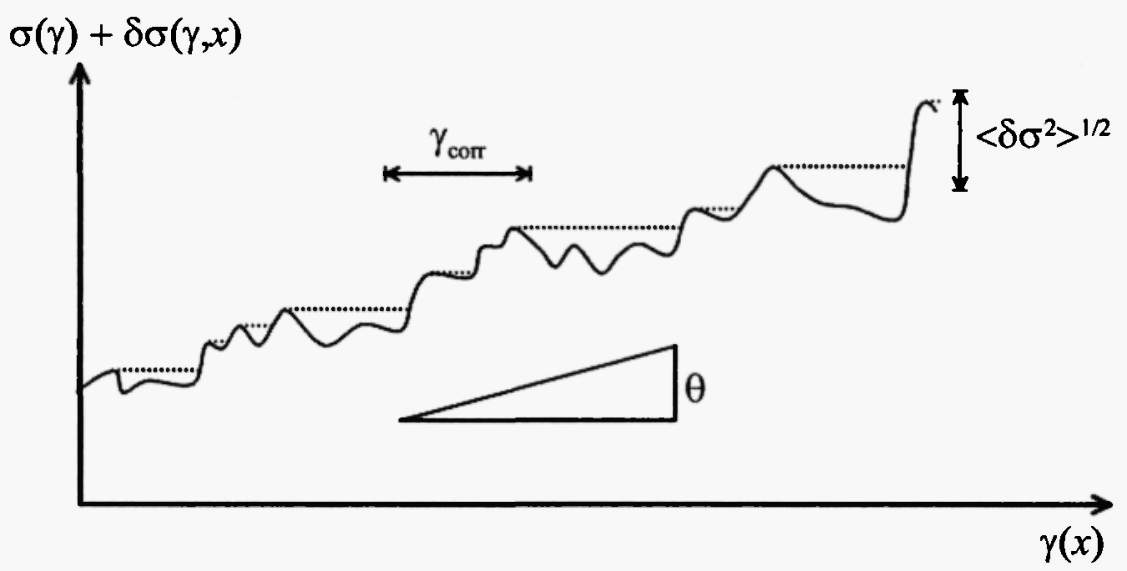

Fig. 4: Local stress-strain characteristics with illustration of some notations introduced in the text: random fluctuations of the flow resistance with amplitude $\left\langle\delta \sigma^{2}\right\rangle$ are superimposed on a linear hardening with slope $\theta$; the dotted lines illustrate the 'jumps' which may occur when the system enters a local softening regime.

In the presence of a non-vanishing gradient term, this picture must be substantially modified since the gradient term leads to stress re-distribution: when the strain increases locally, the flow resistance is increased at this location, while in the vicinity it is decreased - in other words, stress re-distribution takes place. At any time, parts of the specimen are in metastable configurations where a strain jump may take place when the stress is raised slightly. Without the gradient term, this can happen only due to an external stress increase, and accordingly jumps are uncorrelated. When there is a non-zero gradient term, on the other hand, a local strain jump may trigger other jumps in the surrounding. This gives rise to an avalanche-like dynamics, which we will study in the following. Because of this avalanche dynamics, it is not straightforward to claim that on large scales fluctuations average out - and indeed, in certain limit cases they may not average out at all.

\subsection{Characteristic length scales}

To obtain a 'feeling' for the spatial organization of slip which arises when spatial couplings are taken into account, it is useful to start with some qualitative considerations. We consider the non-dimensional version of our constitutive equation, Eq. (5). The gradient term in this equation tends to smooth out deformation heterogeneities. To consider its relative magnitude in comparison with the other terms, we assume that the variable $Y$ varies over the characteristic length $X$ by a unit amount. Then the gradient term is of the order of $1 / X^{2}$. We compare this with the fluctuating stress contribution $\delta \sigma:$ In scaled coordinates, the fluctuations have unit correlation length, and hence their average over the distance $X$ is of magnitude $\left(\left\langle W^{2}>\right| X\right)^{1 / 2}$. Equating this to the magnitude of the gradient term, $1 / X^{2}=\left(\left\langle W^{2}\right\rangle / X\right)^{1 / 2}$, yields a characteristic length $X_{\mathrm{l}}=\left(\left\langle W^{2}\right\rangle\right)^{1 / 3}$. Below this scale, which corresponds to the 'Larkin length' of the theory of depinning, the gradient term always 'wins' against the randomness and keeps the deformation field smooth. On scales above the Larkin length, 
the gradient term still leads to stress redistribution, but at the same time the randomness which comes from the fluctuating deformation resistance is of crucial importance and the deformation field exhibits irregular fluctuations.

A second characteristic scale is obtained by comparing the gradient and hardening terms. We again consider a unit variation of strain over a distance $X$. The variation of the hardening term is of order $H$ while the variation of the gradient term is again of order $1 / X^{2}$. Comparing both terms shows that above a characteristic scale $X_{\mathrm{C}}(H)=H^{-1 / 2}$ the gradient term can be neglected in comparison with the hardening. We will demonstrate below that above this scale the deformation field becomes smooth again.

\subsection{Behaviour in the absence of hardening: depinning, avalanches and self-organized criticality}

We now study the system in the limit where the average hardening is zero, $\theta=H=0$. For $H=0$, Eq. (5) reduces to an equation which has been extensively studied in the context of interface depinning (see e.g. $/ 16,17 /)$. The behaviour of this equation can be summarized as follows: For stresses below a critical (scaled) stress $S_{\mathrm{C}}$, deformation ultimately stops, while above the critical stress the system deforms continually. In the vicinity of the depinning transition which takes place as stress approaches $S_{\mathrm{C}}$, the dynamics of the system and the strain pattern are governed by characteristic power laws:

- Above $S_{\mathrm{C}}$ the average strain rate increases like $\dot{Y} \sim\left(S-S_{\mathrm{C}}\right)^{\beta} . \beta$ is called the velocity exponent

- From the elementary length $X_{\mathrm{L}}$ up to a characteristic scale $X_{\mathrm{C}}$, the strain distribution is self-affine, i.e., the average difference in strain between two points $X$ and $X^{\prime}$ grows like $\left\langle/ Y(X)-Y\left(X^{\prime}\right)^{2}>\sim\left|X-X^{\prime}\right|^{2 \zeta} . \zeta\right.$ is called the roughness exponent.

- The correlation length $X_{\mathrm{C}}$ diverges at the depinning transition like $X_{\mathrm{r}} \sim\left(S-S_{\mathrm{C}}\right)^{-v}$. The exponent $v$ is called the correlation length exponent.

- Deformation proceeds close to the critical point in discrete jumps ('avalanches'). The local strain increment during such an avalanche is $\Delta Y(X)$, and the total strain increment (the avalanche size) $s=$ $(1 / L) \int \Delta Y(X) \mathrm{d} x$. The avalanches exhibit a power-law size distribution,

$$
p(s)=s^{-r} f\left(s / s_{\mathrm{MAX}}\right)
$$

where $\tau$ is called the avalanche exponent and the function $f$ truncates the distribution at a maximum avalanche size $s_{\mathrm{MAX}}$. From the other scaling relations it follows that $s_{\mathrm{MAX}} \sim X_{\mathrm{C}}{ }^{(1+\zeta)} \sim\left(S-S_{\mathrm{C}}\right)^{v(1+\zeta)}$. Close to the critical point, the maximum avalanche size diverges, which implies that there are avalanches on all scales up to the system size. (The minimum avalanche size, on the other hand, is governed by the 'Larkin length', $s_{\mathrm{MIN}} \sim \ddot{x}_{\mathrm{L}}$, which is fixed by the system parameters.) 
The characteristic exponents may be determined either from simulations or from functional renormalization group analysis. Values determined from simulations reported by Leschhorn et al. /17/ for linear interface depinning in 2 dimensions (this corresponds to the case of Eq. (5)) are compiled in Table 1.

\section{Tabie 1}

Scaling exponents characterizing the critical behaviour associated with the depinning transition described by Eq. (5) in the zero-hardening case

\begin{tabular}{|l|l|l|}
\hline Exponent & Scaling relation & Value for Eq. (5) \\
\hline velocity exponent $\beta$ & $\dot{Y} \sim\left(S_{-}-S_{\mathrm{C}}\right)^{\beta}$ & $\beta=0.33$ \\
\hline roughness exponent $\zeta$ & $\langle/ Y(X)-Y(X))^{2}>\sim\left|X-X^{\prime}\right|^{2 \zeta}$ & $\zeta=1.25$ \\
\hline correlation length exponent $v$ & $X_{\mathrm{C}} \sim\left(S_{-}-S_{\mathrm{C}}\right)^{-v}$ & $\nu=1.33$ \\
\hline avalanche exponent $\tau$ & $p(s)=s^{-\tau} f\left(s / s_{0}\right)$ & $\tau=1.05$ \\
\hline
\end{tabular}

In general, to observe the critical behaviour one has to fine tune the stress to the critical value $S_{\mathrm{C}}$. In the present case, however, the stress is not prescribed at a fixed value but results from the machine equation, Eq. (6). To study the resulting dynamics, we have performed simulations of a discrete automaton version of Eq. (5).

In the automaton simulation, space is discretized $\left(X \in\left[X_{1} \ldots X_{N}\right]\right)$ and the gradient term in Eq. (5) is replaced by the discrete gradient. In each 'time step', the strain $Y\left(X_{i}\right)=Y_{i}$ is increased by a discrete amount $\mathrm{d} Y$ when the stress acting on the 'site' $i$ is positive, i.e., when the right-hand side of Eq. (5) is positive. Such sites are called 'active'. After all strains $Y_{i}$ are updated, the total strain is evaluated according to $\{Y\}=(1 / N)$ $\Sigma, Y_{i}$, the externally imposed strain is augmented by an amount $\mathrm{d} Y_{\mathrm{EXT}}$, and new stresses are computed from Eqs. (5) and (6). The same procedure is repeated in the next time step. Different driving rates can be imposed by varying $\mathrm{d} Y_{\mathrm{EXT}}$. Finally, an infinitely slow driving rate can be mimicked by increasing the imposed strain only when there are no active sites, i.e., when the system has come to a rest. This is the typical driving mode for cellular automata exhibiting 'self-organized criticality' $/ 15 /$; in the present context it corresponds to the limit case of a rate-independent material where $\mu=0$.

Figure 5 shows the result of an automaton simulation in the slow-driving limit. It is seen that the plastic response is jerky and proceeds in discrete 'avalanches' of widely varying size, which are separated by quiescent regions where there are no active sites and, hence, the plastic strain does not increase. Each avalanche leads to a sudden increase of the total strain and thereby to a stress drop. The distribution of avalanche sizes (here defined as $N$ times the total strain increment during an avalanche) corresponding to the same simulation is shown in Figure 6. Over three orders of magnitude, the distribution follows a power law with an exponent close to -1 as expected according to Table 1 , while above a critical avalanche size, the probability of large avalanches decays rapidly. 


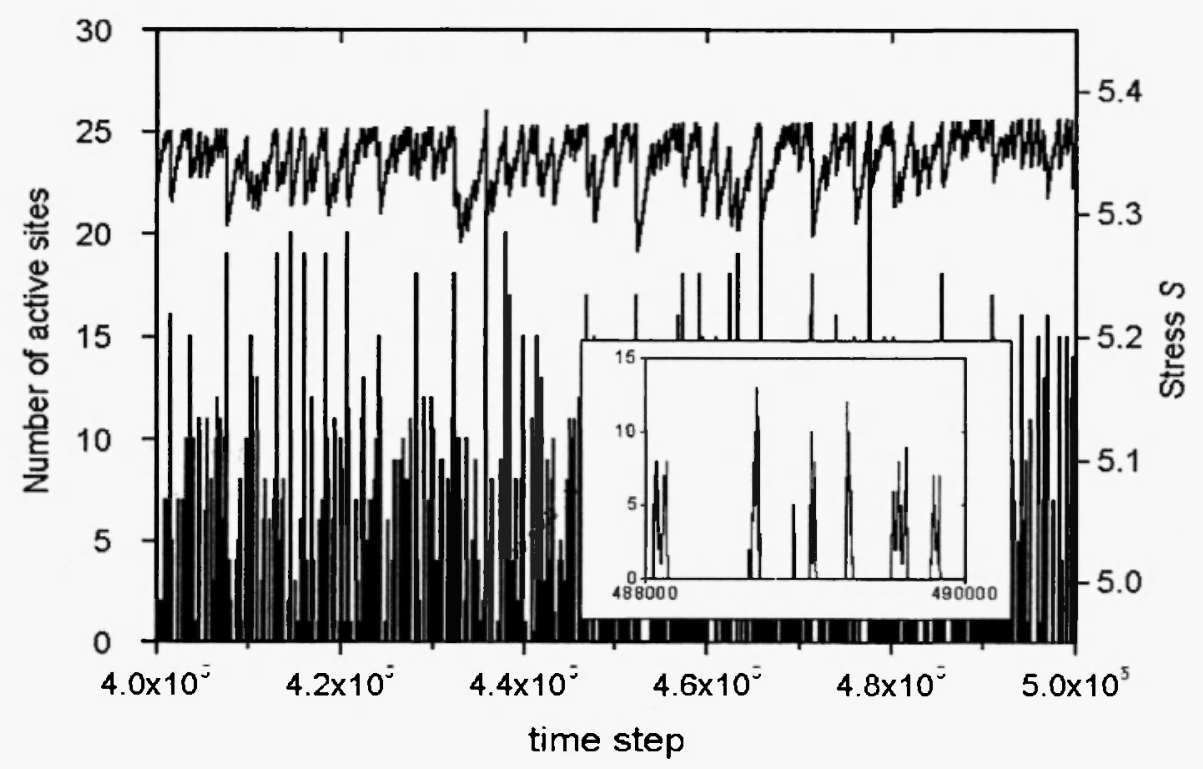

Fig. 5: Simulation of an automaton version of Eqs. (5) and (6) in the limit of zero hardening and small driving rate; parameters: number of sites $N=10000$, yield stress $S_{0}=5$, machine stiffness $M=1 / 2$, external strain increment per time step $\mathrm{d} Y_{\mathrm{EXT}}=10^{-4}$, local strain increment on an active site $\mathrm{d} Y=1$, fluctuation strength $\left\langle W^{2}\right\rangle=1 / 3$, the fluctuations are assumed Gaussian distributed; top curve: stresstime characteristics, bottom curve: 'strain rate' (instantaneous number of active sites per time step); insert: number of active sites vs. time between time steps 48800 and 49000.

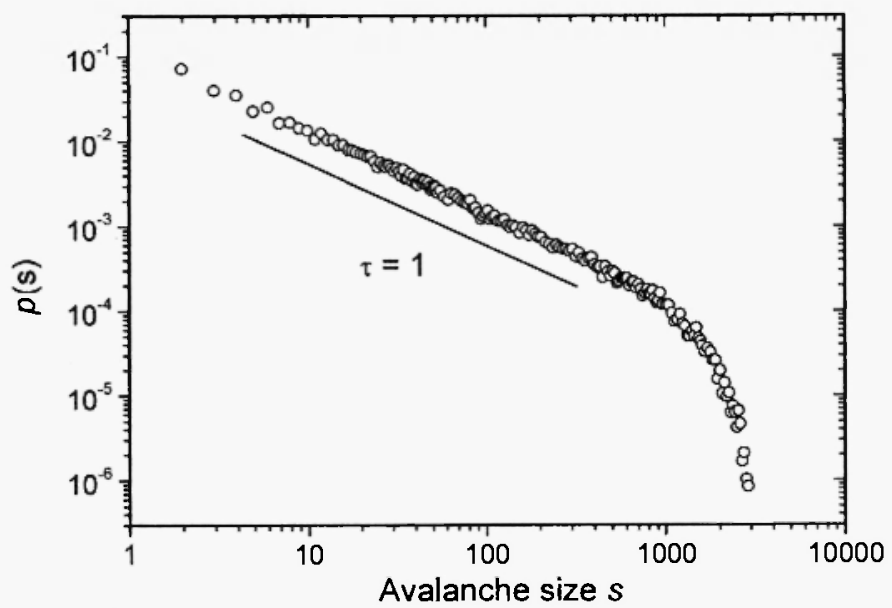

Fig. 6: Avalanche size distribution obtained from automaton simulation for the same parameters as in Fig. 5. 
The parameters of the simulation are chosen such that $X_{\mathrm{L}} \sim 0.7$. Since the smallest avalanche size resolved by the simulation is 1 , the lower cut-off at the Larkin length is not visible in Figure 6 . The origin of the upper cut-off can be understood from the fact that, according to Eq. (6), the strain increase during an avalanche leads to a decrease in stress which is proportional to the avalanche size. This effect leads to a suppression of large avalanches. To validate this conjecture, we note that according to Eq. (6) the stress decrease during an avalanche is expected to be proportional to the ratio $M / L$ (in the actual simulation this is equal to $M / N$ since each 'site' corresponds to a specimen segment of unit scaled length). If the stress decrease is responsible for the truncation, one expects that avalanche size distributions which are obtained for different values of $M$ and $L$ coincide when $s$ is rescaled by some power of $(M / L)$. Figure 7 indicates that this is indeed the case: curves obtained for different $M$ and $N$ coincide when $s$ is rescaled by $(M / N)^{\eta}$ where $\eta \sim 0.75$. (Note that instead of the probability density $p(s)$ we depict the cumulative probability distribution $P(s)=\prod_{s}^{\infty} p(s) \mathrm{d} s$ which evidently contains the same information but can be determined more easily from the simulation.

In the limit where $M$ is zero and/or $N$ is infinite, there is no avalanche cut-off and the system evolves precisely towards its critical state, i.e., it exhibits self-organized criticality. However, it is noted that this behaviour is only observed when several parameters $(M / N, \mu$, and $H)$ are simultaneously tuned to zero.

\subsection{Influence of hardening}

In the presence of hardening, another effect comes into play. In this case, in regions of increased local strain the flow resistance increases locally, and this delimits the magnitude of possible strain 'excursions'.

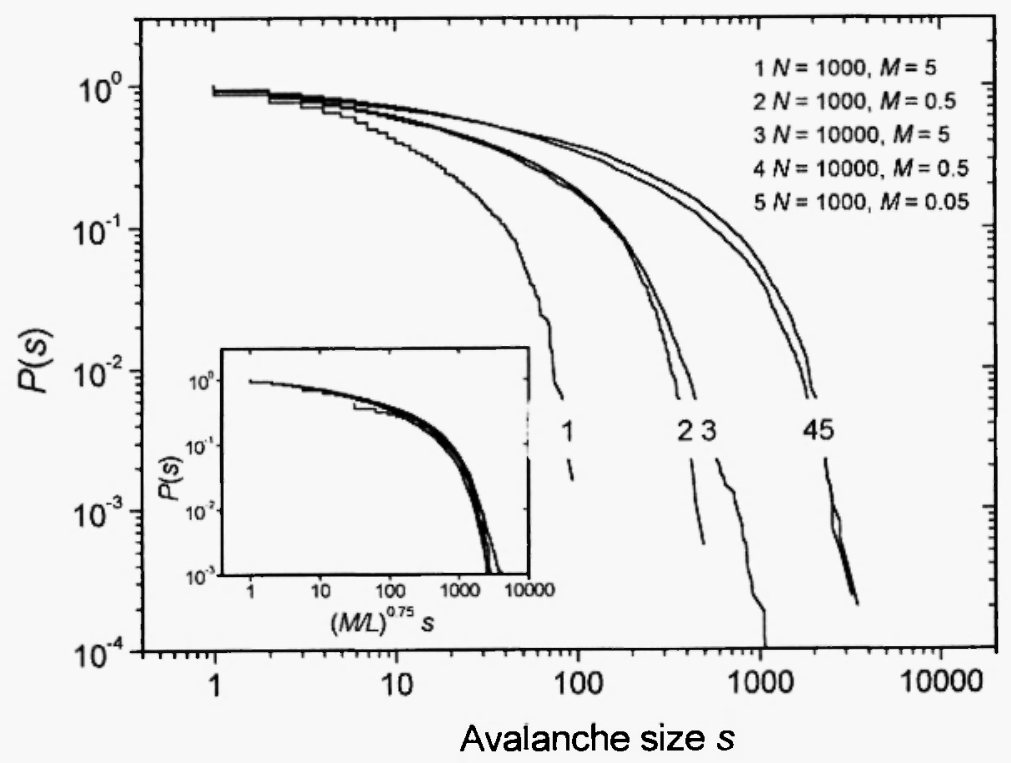

Fig. 7: Cumulative distributions of avalanche sizes for different values of the parameters $N$ and $M$; insert: data collapse obtained after rescaling $s$ by $(M / N)^{0.75}$. 
Accordingly, one expects that avalanches are truncated on the scale $X_{\mathrm{C}}(H)=H^{-1 / 2}$ above which stress redistribution due to the gradient term can be neglected in comparison with the flow-stress increase due to hardening, as discussed in Section 3.2. The corresponding maximum avalanche size is $s_{\mathrm{MAX}} \sim X_{\mathrm{C}}{ }^{(1+\zeta)} \sim H$ $(1+5) / 2$

This is demonstrated in Figure 8 showing cumulative distributions of avalanche sizes obtained for different $H$ and the corresponding data collapse when the avalanche size is rescaled by $H^{(1+5) / 2}$.

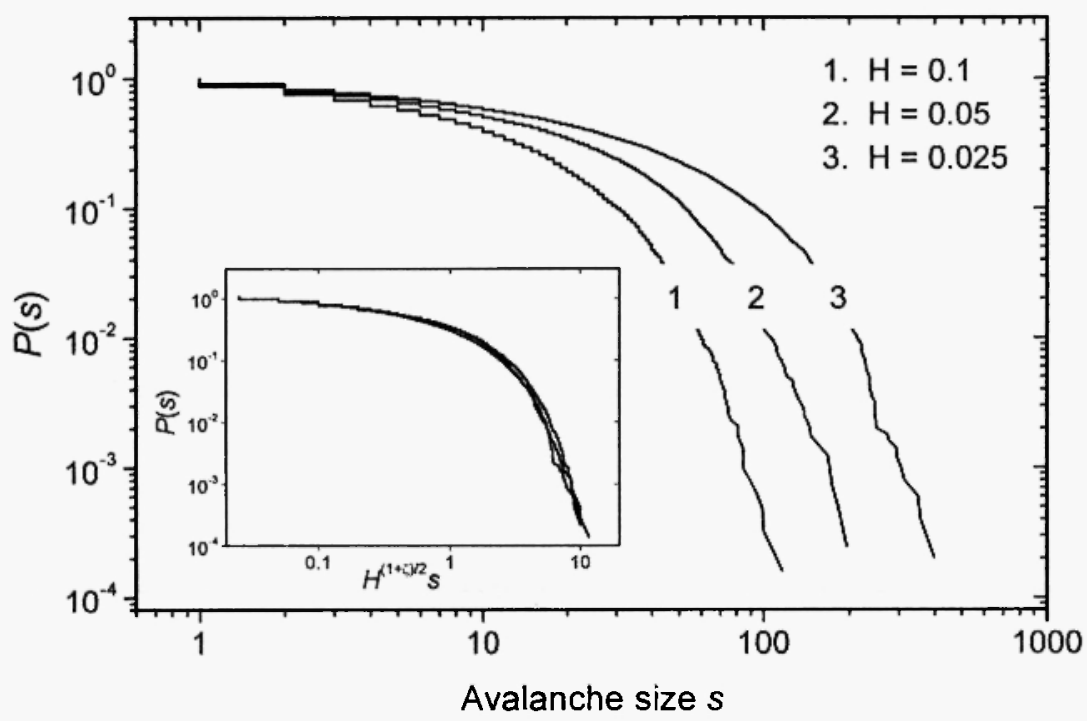

Fig. 8: Cumulative distributions of avalanche sizes for different values of $H$, other parameters as in Figure 5 ; insert: scaling collapse when avalanche size is rescaled by $H^{(1+\zeta) / 2}$.

When hardening is large enough such that $X_{\mathrm{C}}(H)$ is smaller than the 'Larkin length' $X_{\mathrm{L}}$, the avalanche size distribution decreases exponentially on all scales. For $H>1$ (i.e., when $X_{\mathrm{C}}(H)$ falls below the correlation length of the disorder) the system behaves as in the absence of spatial couplings, and there are no avalanches at all.

\subsection{Spatial distribution of strain and surface morphology}

In the regime of scales enclosed by the characteristic lengths $X_{\mathbf{L}}$ and $X_{\mathrm{C}}(H)$, the strain distribution is irregular in space. This is illustrated in Figure 9. The strain distribution shown in the insert of this figure shows an irregular behaviour on small scales; the many sharp peaks of the local strain distribution may, in physical terms, be interpreted as slip lines. 


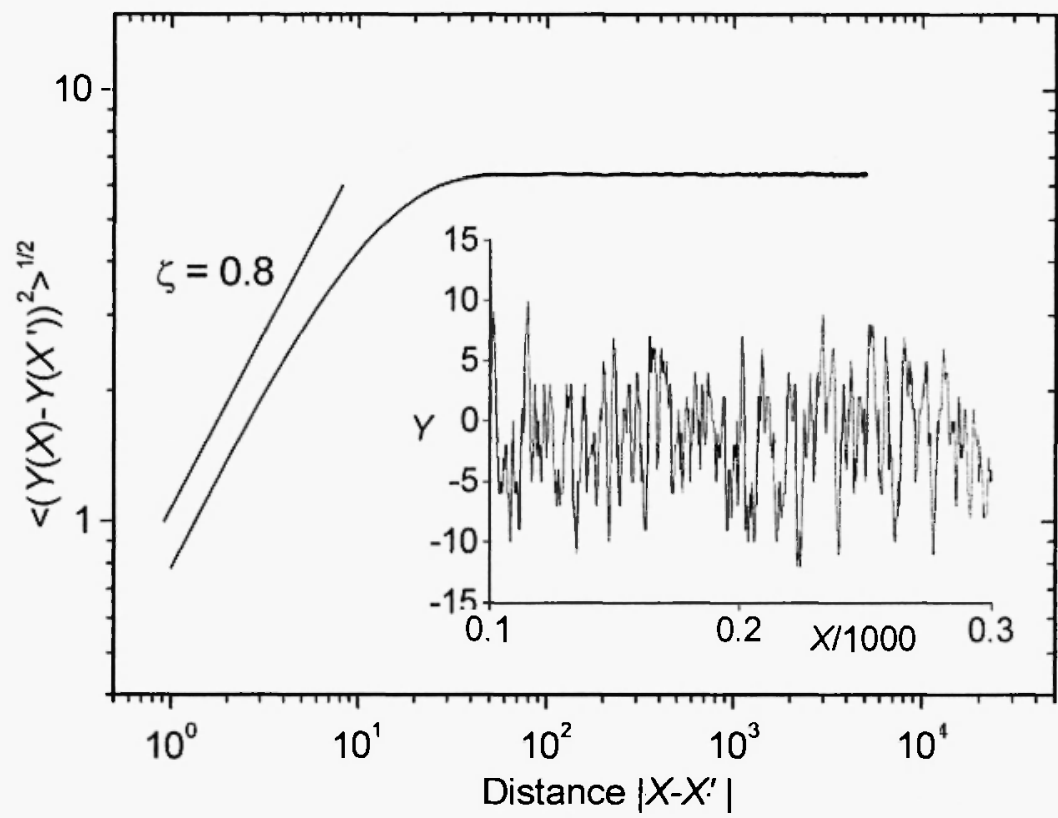

Fig. 9: Statistical characteristics of the spatial distribution of strain; $H=0.01$, other parameters as in Figure 5 ; the curve has been averaged over 50 realizations of the system; insert: spatial strain pofile for one particular realization.

It is seen from Figure 9 that the average strain difference between two points $X$ and $X$ increases for small $\left|X-X^{\prime}\right|$ according to a power law, $\left\langle/ Y(X)-Y\left(X^{\prime}\right)^{2}\right\rangle^{1 / 2} \sim\left|X-X^{\prime}\right|^{\zeta}$. The value of the 'roughness exponent' $\zeta \sim 0.8$ is slightly below the value one expects from Table 1 . On large scales, on the other hand, the strain distribution is perfectly 'flat'. Simulations performed for different values of the hardening coefficient demonstrate that the crossover between both regimes takes place on a characteristic scale which is again proportional to $X_{\mathrm{C}}(H)$, as curves obtained for different $H$ collapse when $X$ is rescaled by $H^{-1 / 2}$.

The profile $Z(X)$ of the surface can be directly obtained from the strain profile since the local strain is the derivative of the surface, $Y(X)=\mathrm{d} Z / \mathrm{d} X$. The insert in Figure 10 shows a surface profile obtained for the same parameters as in Figure 9. It is seen that the surface is rough on all scales. However, statistical analysis of the profile again reveals the presence of two distinct scaling regimes. On small scales, the height difference $\left\langle/ Z(X)-\left.Z(X)\right|^{2}\right\rangle^{1 / 2}$ increases like $\left|X-X^{\prime}\right|^{\zeta}$. with an exponent $\zeta_{s} \sim 0.95$, while on larger scales there is a crossover to $\zeta_{s} \sim 0.5$. Again, simulations for different values of $H$ demonstrate that the crossover length is proportional to the characteristic length $X_{\mathrm{C}}(H)$.

A relation of the type $\left.</ Z(X)-Z(X))^{2}\right\rangle^{1 / 2} \sim\left|X-X^{\prime}\right|^{\zeta}$ implies that the surface profile is self-affine. The fractal dimension $D$ of a self-affine profile is related to its roughness exponent $\zeta_{s}$ by the relation $D=2-\zeta_{s} / 10 /$. Accordingly, the fractal dimension of the surface - which is equal to the dimension $D$ of the surface profile plus one - is close to 2 on small scales and increases to 2.5 on larger scales. 


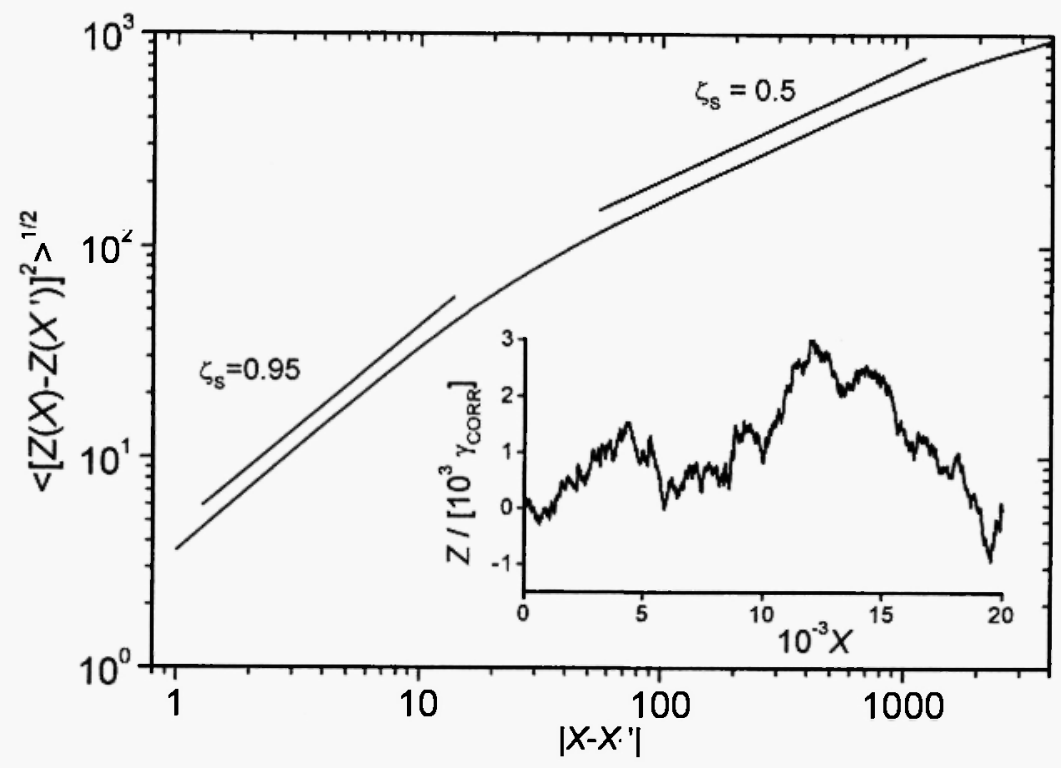

Fig. 10: Surface profile (insert) and roughness exponents of the surface.

\section{DISCUSSION AND CONCLUSIONS}

We have studied a simple gradient constitutive model which includes randomness in terms of an internal stress which is a random function of space and strain. Even in its present, highly simplified version, the model accounts for various spatial and temporal features of deformation in materials where slip proceeds on a single, well-defined plane. We have shown that slip proceeds in avalanches which arise from the stress redistribution by the gradient term in the constitutive equation. The avalanche exponent $\tau \sim 1$ which follows from the present model is, however, smaller than the value of 1.6 reported in the works of Miguel, Weiss et al. /12/. There may be several reasons for this discrepancy: (i) it is not completely clear from $/ 12 /$ whether the quantity measured by Weiss et al. /11/ (the 'energy' of the acoustic wave) is proportional to the strain increment during an avalanche. (ii) The present model has been formulated for an idealized one-dimensional system. In a more realistic model, also heterogeneities and interactions in the plane of slip must be taken into account. This may affect the avalanche exponent for two reasons: (i) The avalanche exponent is known to increase as the dimension of the system increases towards the critical dimension, which is four. For dimensions equal or larger than this value, the avalanche exponent has the mean-field value $\tau=1.5$ which is close to the value of Weiss et al. (ii) Interactions which arise from heterogeneities of slip in the slip plane have long-range character. Systems with long-range interactions, however, may exhibit mean-field behaviour even for dimensions equal or less than three $/ 17 /$. To include these interactions and generalize the model to 
three dimensions requires, however, additional considerations since in this case the tensorial nature of the stress and strain fields must be taken into account. Such an investigation, which is of obvious relevance for the plastic behaviour of random media in general, is postponed to future work.

Even in its present simplified form, the model accounts for many features of the spatial distribution of slip and the surface morphology of crystals deforming on a single slip system. The model leads to an erratic distribution of slip, characterized by sharp peaks which may be interpreted as slip lines. The magnitude of these peaks is, however, limited and the slip distribution is more or less homogeneous on large scales. The surface morphology is rough and characterized by two scaling regimes with different roughness exponents. These exponents correspond to fractal dimensions close to 2 on small and close to 2.5 on large scales. The crossover between both regimes depends on the hardening coefficient and occurs already on small scales when the hardening is high. Also this finding is consistent with experimental observations: Meissner et al. /9/ report a fractal dimension of the surface which is close to 2 in hardening Stage I (easy glide) where the hardening coefficient is low, and an abrupt increase of the fractal dimension to 2.5 in hardening Stage II where the hardening coefficient is about one order of magnitude larger.

The present results have been obtained in a rather simple setting which underlines the analogy with problems that have been studied in the physics of random or disordered systems $/ 17-19 /$. We expect. however, that the basic features which we observe may be much more general and appear in many situations where disordered materials undergo plastic deformation. Finally, we emphasize that the present notion of 'disorder' even applies to crystalline materials when the plastic flow resistance is governed by the existence of a random microstructure.

\section{ACKNOWLEDGEMENTS}

The authors acknowledge support by the Commission of the European Communities under the RTN DEFINO project No. HPRN-CT-2002-00198 and RTN-DIGA project No. HPRN-CT-2002-00220.

\section{REFERENCES}

1. E.C. Aifantis. On the microstructural origin of certain inelastic models, Transactions of the ASME, Journal of Engineering Materials and Technology, 106(4), 326-330 (1984).

2. E.C. Aifantis. The physics of plastic deformation, Int. J. Plasticity 3, $211-247$ (1987).

3. H.M. Zbib and E.C. Aifantis. On the localization and postlocalization behavior of plastic deformation, Res Mechanica 23, 261-305 (1988).

4. L.P. Kubin, Y. Estrin and E.C. Aifantis. Introductory remarks to the viewpoint set on propagative plastic instabilities, Scripta Metallurgica et Materialia 29, 1147-1150 (1993). 
5. H. Neuhäuser. Slip-line formation and collective dislocation motion, in: Dislocations in Solids Vol. 4, F.R.N. Nabarro (Ed.), North-Holland, Amsterdam 1984, p. 319.

6. B. Sprusil and F. Hnilica. Fractal character of slip lines of Cd single crystals, Czech. J. Phys. B 35, 897900 (1985).

7. T. Kleiser and M. Bocek. The fractal nature of slip in crystals, Z. Metallkde. 77, 582-587 (1986).

8. H. Neuhäuser. The dynamics of slip-band formation in single crystals, Res Mechanica 23, 113-135 (1988).

9. O. Meißner, J. Schreiber and A. Schwab. Formation of mesostructures at the surface of ferritic steel and a nickel monocrystal under increasing load - An in-situ AFM experiment, Appl. Phys. A 66, S $1113-$ S1116 (1998).

10. E. Bouchaud. Scaling properties of cracks, J. Phys.-Condens. Mat. 9, 4319-4344 (1997).

11. J. Weiss and J.-R. Grasso. Acoustic emission in single crystals of ice, J. Phys. Chem. B 32, 6113-6117 (1997).

12. M.-C. Miguel, A. Vespignani, S. Zapperi, J. Weiss and J.-R. Grasso. Intermittent dislocation flow in viscoplastic deformation, Nature 410, 667-671 (2001).

13. P. Hähner. On the foundations of stochastic dislocation dynamics, Appl. Phys. A 64, 473-481 (1996).

14. P. Hähner and M. Zaiser. From mesoscopic fluctuations of slip to macroscopic fluctuations of stress and strain, Acta Materialia 45, 1067-1075 (1997).

15. P. Bak, C. Tang and K. Wiesenfeld. Self-organized criticality - an explanation for $1 / \mathrm{f}$ noise, Phys. Rev. Letters 59, 381-384 (1987).

16. M. Zaiser, I. Groma and E.C. Aifantis, forthcoming.

17. S. Zapperi, P. Cizeau, G. Durin and H.E. Stanley. Dynamics of a ferromagnetic domain wall avalanches, depinning and the Barkhausen effect, Phys. Rev. B 58, 6353-6366 (1998)

18. M. Kardar. Non-equilibrium dynamics of interfaces and lines, Phys. Rep. 301, 85-112 (1998).

19. H. Leschhorn, T. Nattermann, S. Stepanow and L.H. Tang. Driven interface depinning in a disordered medium, Ann. Phys. 6, 1-34 (1997). 